\title{
Modalidades de aplicação e associações de herbicidas no controle de plantas daninhas em milho em espaçamento convencional e reduzido
}

\author{
Modalities of application and tank-mix combinations of herbicides \\ on corn under conventional and narrowed spacing
}

\author{
Antonio Mendes de Oliveira Neto ${ }^{1 *}$; Rubem Silvério de Oliveira Júnior ${ }^{2}$; \\ Jamil Constantin'; ${ }^{3}$ Diego Gonçalves Alonso ${ }^{3}$; Michel Alex Raimondi'; \\ Gizelly Santos ${ }^{2}$; Alexandre Gemelli ${ }^{4}$
}

\section{Resumo}

\begin{abstract}
A redução de espaçamento entre linhas é uma prática que favorece a cultura do milho na competição com as plantas daninhas, no entanto, pouco se sabe sobre o uso de herbicidas para o controle de plantas daninhas nestas condições, portanto, objetivou-se nesta pesquisa avaliar a eficiência e seletividade de diferentes combinações de herbicidas e modalidades de aplicação em pós-emergência sobre o controle de plantas daninhas na cultura do milho conduzida em espaçamento convencional e reduzido. Os tratamentos avaliados foram as associações entre os herbicidas atrazine+nicosulfuron+mesotrione, atrazine + nicosulfuron, atrazine + mesotrione e nicosulfuron + mesotrione aplicados em modalidade única e sequencial mais duas testemunhas (uma capinada e outra sem capina). De forma geral, os tratamentos herbicidas foram seletivos a cultura do milho e não afetaram significativamente os componentes de produção e o rendimento de grãos. Os resultados de controle para modalidade de aplicação sequencial mantiveram valores de controle satisfatório, para a maioria dos tratamentos, até a pré-colheita da cultura do milho, sendo um indício que esta modalidade de aplicação apresenta um período de controle efetivo maior para as misturas em tanque avaliadas.
\end{abstract}

Palavras-chave: Aplicação seqüencial, eficiência, seletividade, Zea mays

\begin{abstract}
Narrowing the distance between sowing lines is a practice that may help corn crop as a competitor against weeds, but little is known about weed chemical control under such conditions. Therefore, the objective of this research was to evaluate the efficiency of different combinations of herbicides and modalities of post-emergence application on weed control in corn cultivated under conventional $(0.90 \mathrm{~m})$ and narrow $(0.45 \mathrm{~m})$ spacing between rows. Treatments evaluated included the association of atrazine+nicosulfur on + mesotrione, atrazine + nicosulfuron, atrazine + mesotrione and nicosulfuron + mesotrione, applied in unique or sequential application, and controls (handweeded control and without handweeded control). In general all treatments were considered as selective for corn and did not affect yield components and
\end{abstract}

\footnotetext{
${ }^{1}$ Eng $^{\circ}$ Agr $^{\circ}$, Mestrando do Programa de Pós-graduação em Agronomia da Universidade Estadual de Maringá, NAPD/UEM, Bolsista Capes. E-mail: am.oliveiraneto@hotmail.com; gizelly@agronoma.eng.br

${ }^{2}$ Eng $^{\mathrm{o}}$ Agr $^{\mathrm{o}}$, D.Sc. Professor Associado, Coordenador do Núcleo de Estudos Avançados em Ciência das Plantas Daninhas, NAPD/ UEM, Departamento de Agronomia, Universidade Estadual de Maringá, UEM. Bolsista CNPq. E-mail: rsojunior@uem.br; constantin@teracom.com.br

${ }^{3}$ Eng $^{\circ}$ Agro , M.Sc., Doutorando do Programa de Pós-graduação em Agronomia da Universidade Estadual de Maringá, NAPD/ UEM, Bolsista CNPq. E-mail: alonsodg07@hotmail.com; michelraimondi@hotmail.com

${ }^{4}$ Discente do curso de Agronomia da Universidade Estadual de Maringá, NAPD/UEM. Bolsista CNPq. E-mail: alexandregemelli@ hotmail.com

* Autor para correspondência
} 
grain yield. Results concerning weed control for sequential applications were considered as satisfactory in most treatments up to corn harvest, what was considered as an indicative that such modality presents a longer effective period of weed control considering the evaluated treatments.

Key words: Sequential application, efficiency, seletictivity, Zea mays

\section{Introdução}

As condições edafoclimáticas destacam o Brasil como um país de grande potencial para a cultura do milho. No entanto, o clima tropical é também favorável à ocorrência de uma grande quantidade de plantas daninhas, que interfere no desenvolvimento e na produtividade da cultura (CARVALHO; PERUCHI; PALAZZO, 2001).

As plantas daninhas interferem no desenvolvimento da cultura do milho com intensidade variável em função da época de ocorrência, da população e das espécies presentes. A ocorrência de uma elevada infestação no início do desenvolvimento da cultura pode proporcionar perdas acentuadas na produtividade se o controle e a época de aplicação não forem adequados (ZAGONEL; VENÂNCIO; KUNZ, 2000; GALON et al., 2008). Estima-se que a redução de rendimento em função da competição com as plantas daninhas sejam da ordem de 13\%, porém, em muitas situações onde nenhuma medida de controle é adotada, essa redução pode chegar a $85 \%$ (CARVALHO et al., 2007), o que torna o controle de plantas daninhas uma necessidade de ordem econômica (LÓPEZOVEJERO et al., 2003).

Alterações nas relações de competição por água, luz e nutrientes entre o milho e as plantas daninhas, em favor da cultura, podem ser alcançadas por meio de práticas de manejo, como por exemplo, a mudança no espaçamento entre fileiras que alterará o arranjo espacial das plantas na área (BALBINOT JÚNIOR; FLECK, 2004), além disso, os arranjos espaciais mais eqüidistantes promovem menor competição intraespecífica, favorecendo a cultura na competição interespecífica (NICE; BUEHRING; SHAW, 2001). Na cultura do milho a tendência atual é de reduzir o espaçamento entre linhas, de forma a modificar o comportamento da planta e interferir na eficiência de utilização dos recursos do meio, mantendo a densidade de plantas constante (DEMÉTRIO et al., 2008).

Mesmo que a redução no espaçamento entrelinhas não resulte necessariamente em aumento do rendimento de grãos (STRIEDER et al., 2008), sua adoção justifica-se pelo aumento da competitividade da cultura com as plantas daninhas, devido à maior quantidade de luz que é interceptada pelo dossel, podendo reduzir a dependência de herbicidas (TEASDALE, 1995).

O controle químico de plantas daninhas geralmente possibilita uma garantia de produção para a cultura, devido à eliminação da competição de plantas daninhas por recursos do meio. Ainda que o uso de herbicida cause sintomas de fitointoxicação à cultura, o rendimento pode ser alto devido à eficiência no controle das plantas daninhas (MÔRO; DAMIÃO FILHO, 1999).

A adoção da aplicação sequencial de herbicidas vem se mostrando uma alternativa interessante para o controle de plantas daninhas, já que o parcelamento da dose acaba aumentando a seletividade dos herbicidas sobre as culturas (OLIVEIRA JÚNIOR et al., 2006b) e aumenta a eficiência do controle. Burke et al. (2008) relataram que a aplicação sequencial de nicosulfuron em pós-emergência da cultura do milho foi mais eficiente no controle de plantas daninhas que a aplicação única. A aplicação sequencial do herbicida flumiclorac-pentil em pós-emergência precoce de plantas de Euphorbia heterophylla apresentou bons resultados de controle em condições de campo e casa-de-vegetação (OLIVEIRA JÚNIOR et al., 2006b). Constantin et al. (2007), relataram que a aplicação sequencial de flumiclorac-pentil foi mais eficiente no controle de diferentes espécies de guanxuma comparado a aplicação em dose única. 
O objetivo deste trabalho foi avaliar a eficiência e seletividade de diferentes combinações de herbicidas e modalidades de aplicação em pósemergência sobre o controle de plantas daninhas na cultura do milho.

\section{Material e Métodos}

Dois experimentos foram conduzidos simultaneamente em condições de campo durante a safra de verão 2008/2009, em área de semeadura direta, pertencente à Fazenda Experimental de Iguatemi (S 2320'e W 052 04 ', à $508 \mathrm{~m}$ de altitude), localizada no distrito de Iguatemi, município de Maringá, PR. No primeiro experimento a cultura do milho foi conduzida no espaçamento convencional de $0,90 \mathrm{~m}$ entre linhas e no segundo foi conduzido em espaçamento reduzido entre linhas de $0,45 \mathrm{~m}$. $\mathrm{O}$ solo da área experimental foi classificado como Latossolo Vermelho distroférrico (EMBRAPA, 1999; ARAÚJO; TORMENA; SILVA, 2004).

A análise química da camada superficial (0 a $20 \mathrm{~cm}$ ) na área conduzida sob espaçamento convencional $(0,90 \mathrm{~m})$ apresentou as seguintes características: $\mathrm{pH}$ em água de 4,90; 3,97 cmolc $\mathrm{dm}^{-}$ ${ }^{3} \mathrm{de} \mathrm{H}^{+}+\mathrm{Al}^{+3}$; 1,43 cmolc dm ${ }^{-3}$ de $\mathrm{Ca}^{+2} ; 0,34$ cmolc $\mathrm{dm}^{-3}$ de $\mathrm{Mg}^{+2} ; 0,27$ cmolc $\mathrm{dm}^{-3}$ de $\mathrm{K}^{+} ; 33,35 \mathrm{mg}$ $\mathrm{dm}^{-3}$ de P; 5,44 $\mathrm{g} \mathrm{dm}^{-3}$ de C; constituído por $29 \%$ de areia grossa; $49 \%$ de areia fina; $4 \%$ de silte e $18 \%$ de argila. Já a análise química da área cultivada sob espaçamento reduzido $(0,45 \mathrm{~m})$ apresentou as seguintes características: $\mathrm{pH}$ em água de 5,30; 3,30 cmolc $\mathrm{dm}^{-3} \mathrm{de}^{+}+\mathrm{Al}^{+3} ; 1,45$ cmolc $\mathrm{dm}^{-3}$ de $\mathrm{Ca}^{+2}$; 0,43 cmolc dm ${ }^{-3}$ de $\mathrm{Mg}^{+2} ; 0,20$ cmolc $\mathrm{dm}^{-3}$ de $\mathrm{K}^{+}$; 25,25 mg dm ${ }^{-3}$ de P; $6,17 \mathrm{~g} \mathrm{dm}^{-3}$ de C; constituído por $32 \%$ de areia grossa; $52 \%$ de areia fina; $2 \%$ de silte e $14 \%$ de argila.

Antes da instalação da cultura do milho, a área foi cultivada com aveia (Avena sativa), a qual foi dessecada com glyphosate (900 g e.a. ha-1) 12 dias antes da semeadura do milho. A adubação de base utilizada na área foi de $220 \mathrm{~kg} \mathrm{ha}^{-1}$ da formulação 04-20-20. Aos 25 dias após a semeadura realizou- se a adubação de cobertura com $80 \mathrm{~kg} \mathrm{ha}^{-1}$ de uréia, sendo que nesta ocasião as plantas estavam no estádio fenológico de V6. A semeadura direta do milho ocorreu em 17/12/2008. No primeiro experimento o espaçamento entre linhas adotado foi de $0,90 \mathrm{~m}$ e uma densidade de semeadura de aproximadamente 7,0 sementes por metro linear. No segundo experimento adotou-se o espaçamento entre linhas de $0,45 \mathrm{~m}$ e a densidade de semeadura de aproximadamente 3,5 sementes. Em ambos os experimentos o híbrido utilizado foi o híbrido simples 30K75Y.

O tratamento de sementes foi realizado com o inseticida Imidacloprid + Thiodicarb $(150+450$ g i.a. para cada $100 \mathrm{~kg}$ de sementes). Durante o ciclo do milho, foram realizadas três aplicações de inseticidas para o controle de Spodoptera frugiperda (lagarta-do-cartucho), sendo a primeira realizada com o inseticida novarulon (15 g i.a. ha $\left.{ }^{-1}\right)$ e as demais com novarulon ( 15 g i.a. ha $\left.{ }^{-1}\right)+$ metomil $\left(172\right.$ g i.a. ha $\left.{ }^{-1}\right)$.

O delineamento utilizado foi o de blocos ao acaso, com 10 tratamentos e quatro repetições, sendo as parcelas de 5,0 x 5,0 $\mathrm{m}\left(25,0 \mathrm{~m}^{2}\right)$. Considerou-se como área útil para as avaliações apenas as linhas centrais de cada parcela, exceto $0,5 \mathrm{~m}$ de cada extremidade, totalizando uma área útil de $16 \mathrm{~m}^{2}$. Entre os blocos foi deixado espaço de um metro, o qual serviu como bordadura adicional.

Os tratamentos consistiram da aplicação dos herbicidas em duas modalidades distintas (Tabela 1), uma aplicação única em pós-emergência realizada no dia 06/01/2009 (20 dias após a semeadura), com as plantas de milho entre os estádios fenológico V4 e V5. A segunda modalidade de aplicação foi sequencial onde os tratamentos foram parcelados em dosagens iguais em duas épocas distintas de aplicação, a primeira foi realizada no dia 30/12/2008 (13 dias após a semeadura) e a cultura no estádio fenológico de V2, a segunda aplicação foi realizada no dia 06/01/2009 (20 dias após a semeadura) com a cultura entre os estádios de V4 e V5. 
Para o espaçamento convencional, no momento da primeira aplicação sequencial as plantas de Digitaria horizontalis encontravam-se no estádio de 2 a 4 folhas e densidade de 28 plantas por $\mathrm{m}^{2}$, e as condições no momento da aplicação foram de $\mathrm{T}=29^{\circ} \mathrm{C}, \mathrm{UR} \%=65 \%$, ventos máximos de $4 \mathrm{~km} \mathrm{~h}^{-1}$, solo seco e céu claro e sem nuvens. $\mathrm{Na}$ segunda aplicação, as plantas estavam com 2 a 4 folhas e algumas plantas remanescentes em início de perfilhamento, na densidade de 22 plantas por $\mathrm{m}^{2},\left(\mathrm{~T}=24^{\circ} \mathrm{C}, \mathrm{UR} \%=60 \%\right.$, ventos máximos de 4 $\mathrm{km} \mathrm{h}^{-1}$, solo úmido e céu claro e sem nuvens). Para a modalidade de aplicação única, as condições climáticas foram idênticas às descritas para a segunda aplicação sequencial, no entanto, as plantas de $D$. horizontalis estavam no estádio de 2 folhas a 1-2 perfilhos e apresentaram densidade de 12 plantas por $\mathrm{m}^{2}$.

Para o espaçamento reduzido no momento da primeira aplicação sequencial as plantas de $D$. horizontalis estavam no estádio de 2 a 4 folhas e densidade de 25 plantas por $\mathrm{m}^{2},\left(\mathrm{~T}=28^{\circ} \mathrm{C}, \mathrm{UR}=72 \%\right.$, ventos máximos de $3 \mathrm{~km} \mathrm{~h}^{-1}$, solo seco e céu claro e sem nuvens). Na segunda aplicação as plantas de D. horizontalis estavam com 2 a 4 folhas e algumas plantas remanescentes em início de perfilhamento na densidade de 13 plantas por $\mathrm{m}^{2},\left(\mathrm{~T}=24^{\circ} \mathrm{C}\right.$, $\mathrm{UR}=60 \%$, ventos máximo de $5 \mathrm{~km} \mathrm{~h}^{-1}$, solo úmido e céu claro e sem nuvens). Na modalidade de aplicação única as condições climáticas foram idênticas as descritas para a segunda aplicação sequencial, no entanto, as plantas de $D$. horizontalis estavam no estádio de 2 folhas a 1-2 perfilhos e densidade de 16 plantas por $\mathrm{m}^{2}$.

$\mathrm{Na}$ área havia outras espécies de plantas daninhas como Cenchrus echinathus, Raphanus raphanistrum e Commelina benghalensis, no entanto, em menor freqüência e densidade, e com distribuição desuniforme na área experimental.

Para todas as aplicações foi utilizado um pulverizador costal sob pressão constante à base de $\mathrm{CO}_{2}$, equipado com cinco pontas tipo leque XR 110.02 , espaçadas em $50 \mathrm{~cm}$ entre si, operando a pressão de 2,0 $\mathrm{kgf} \mathrm{cm}^{-2}$, que proporcionou o equivalente a $200 \mathrm{~L} \mathrm{ha}^{-1}$ de volume de calda.

Tabela 1. Tratamentos e respectivas doses utilizadas no experimento visando avaliar a eficiência e a seletividade de diferentes modalidades de aplicação de herbicidas na cultura do milho conduzida em espaçamento convencional e reduzido.

\begin{tabular}{|c|c|c|c|}
\hline Tratamentos $^{1}$ & Dose $\left(\mathrm{g} \mathrm{ha}^{-1}\right)$ & $\begin{array}{l}\text { Estádio da } \\
\text { cultura }\end{array}$ & $\begin{array}{c}\text { Modalidade de } \\
\text { aplicação }\end{array}$ \\
\hline 1.atrazine + nicosulfuron + mesotrione $(\mathrm{A}+\mathrm{N}+\mathrm{M})$ & $750+21+85,92$ & V4 a V5 & Única \\
\hline 2. atrazine+nicosulfuron $(\mathrm{A}+\mathrm{N})$ & $1250+18,8$ & V4 a V5 & Única \\
\hline 3. atrazine + mesotrione $(\mathrm{A}+\mathrm{M})$ & $1250+120$ & V4 a V5 & Única \\
\hline 4.nicosulfuron + mesotrione $(\mathrm{N}+\mathrm{M})$ & $21+85,92$ & V4 a V5 & Única \\
\hline 5.atrazine+nicosulfuron+mesotrione $(\mathrm{A}+\mathrm{N}+\mathrm{M})$ & $375+12,75+51,84$ & $\mathrm{~V} 2$ e V4 a V5 & Sequencial \\
\hline 6.atrazine+nicosulfuron $(\mathrm{A}+\mathrm{N})$ & $1250+10$ & $\mathrm{~V} 2$ e V4 a V5 & Sequencial \\
\hline 7.atrazine + mesotrione $(\mathrm{A}+\mathrm{M})$ & $625+60$ & V2 e V4 a V5 & Sequencial \\
\hline 8.nicosulfuron+mesotrione $(\mathrm{N}+\mathrm{M})$ & $12,75+51,84$ & V2 e V4 a V5 & Sequencial \\
\hline 9.testemunha sem capina & - & - & - \\
\hline 10.testemunha capinada & - & - & - \\
\hline
\end{tabular}

${ }^{/ 1}$ Acrescentou-se o adjuvante Joint oil ${ }^{\circledR}$ a $0,5 \% \mathrm{v} / \mathrm{v}$ em todos os tratamentos herbicidas.

Herbicidas unidos por "+” foram aplicados na forma de associação. 
As características avaliadas foram: porcentagem de controle de D. horizontalis (escala visual, 0-100\%, onde $0 \%$ significa ausência de sintomas e 100\% controle total das plantas daninhas) aos 26, 36, $56 \mathrm{e}$ 112 (pré-colheita) dias após a semeadura (DAS) da cultura (SBCPD, 1995), ou seja, 39, 49, 69, 125 dias após a aplicação (DAA) para a aplicação única e 46, 56, 76 e 132 DAA para aplicação sequencial. Na fase de pré-colheita da cultura (112 DAS) realizouse uma avaliação de controle geral, considerando o controle total de plantas daninhas presentes nas parcelas, comparativamente com a testemunha que não recebeu nenhum método de controle $(0 \%$ de controle) e com a testemunha capinada durante todo o ciclo (100\% de controle). As notas de fitointoxicação da cultura foram avaliadas por meio da escala EWRC ( 1 - 9, onde 1 significa ausência de sintomas e 9 controle total das plantas), aos 26, 36, 56 e 112 DAS da cultura (EWRC, 1964). Por ocasião da colheita do milho (17/04/2009), avaliouse a produtividade da cultura (colheita das espigas de todas as plantas da área útil das parcelas - 16,0 $\mathrm{m}^{2}$ ), o número de espigas por parcela, o peso médio das espigas e a razão massa dos grãos/massa total das espigas. Os dados relacionados a produtividade de grãos foram convertidos para $13 \%$ de umidade.
Os resultados de cada experimento foram analisados de maneira separada por meio de análise de variância pelo teste $\mathrm{F}$ e as médias comparadas pelo teste de agrupamento de médias de Scott-Knott (SCOTT; KNOTT, 1974), a 5\% de probabilidade.

\section{Resultados e Discussão}

Os sintomas visuais de fitointoxicação causados pela aplicação das diferentes tratamentos foram observados somente na avaliação de 26 dias após a semeadura (26 DAS) (Tabela 2) e sua intensidade foi variável em função dos herbicidas e das modalidades de aplicação. De forma geral, os tratamentos aplicados na forma sequencial foram mais seletivos as plantas de milho do que a aplicação única, independentemente do espaçamento entre linhas utilizado. Para Oliveira Júnior et al. (2006a) na modalidade de aplicação sequencial ocorre o parcelamento da dose o que acaba na maioria das vezes aumentando a seletividade dos herbicidas. Estes resultados também estão de acordo com os obtidos na aplicação sequencial de herbicidas na cultura da cebola (FERREIRA; DURIGAN; CHURATA-MASCA, 1999, FERREIRA et al., 2000), no consórcio de café e feijão (RONCHI; SILVA, 2004) e na cultura da soja (OLIVEIRA JÚNIOR et al., 2006b).

Tabela 2. Avaliação de fitointoxicação realizada aos 26 dias após a semeadura (DAS) da cultura do milho conduzida em espaçamento convencional (0,90 m) e reduzido (0,45 m). Maringá, PR, 2008/2009.

\begin{tabular}{|c|c|c|}
\hline \multirow{2}{*}{ Tratamentos } & \multicolumn{2}{|c|}{ Fitointoxicação (Escala EWRC*) } \\
\hline & $0,45 \mathrm{~m}$ & $0,90 \mathrm{~m}$ \\
\hline $1 . \mathrm{A}+\mathrm{N}+\mathrm{M}$ & 5,0 & 5,0 \\
\hline 2. $\mathrm{A}+\mathrm{N}$ & 5,0 & 5,0 \\
\hline 3. $\mathrm{A}+\mathrm{M}$ & 5,0 & 5,0 \\
\hline 4. $\mathrm{N}+\mathrm{M}$ & 2,0 & 2,0 \\
\hline Média & 4,25 & 4,25 \\
\hline $5 . \mathrm{A}+\mathrm{N}+\mathrm{M} / \mathrm{A}+\mathrm{N}+\mathrm{M}$ & 2,8 & 4,2 \\
\hline $6 . \mathrm{A}+\mathrm{N} / \mathrm{A}+\mathrm{N}$ & 3,5 & 4,2 \\
\hline 7. $\mathrm{A}+\mathrm{M} / \mathrm{A}+\mathrm{M}$ & 5,0 & 5,0 \\
\hline 8.N+M/N+M & 2,0 & 2,0 \\
\hline Média & 3,33 & 3,85 \\
\hline 9.testemunha sem capina & 1,0 & 1,0 \\
\hline 10.testemunha capinada & 1,0 & 1,0 \\
\hline
\end{tabular}

Obs: $\mathrm{A}=$ atrazine, $\mathrm{N}=$ nicosulfuron, $\mathrm{M}=$ mesotrione.

*Escala EWRC. , onde 1,0 = ausência de sintomas e 9,0 = morte de 100\% das plantas. 
A mistura em tanque de atrazine com mesotrione foi o tratamento que provocou os sintomas mais intensos de fitointoxicação e recebeu nota 5 (Escala EWRC), tanto para o espaçamento convencional como para o reduzido, assim como na aplicação única e na sequencial. Em contrapartida, a combinação entre os herbicidas nicosulfuron e mesotrione foi o único tratamento que recebeu nota 2 de fitointoxicação aos 26 DAS em todas as situações avaliadas.

Os sintomas de fitointoxicação causados pelos tratamentos foram os mesmos tanto no espaçamento convencional como no espaçamento reduzido. A mistura de atrazine + nicosulfuron + mesotrione provocou sintomas que se caracterizaram por necrose nos bordos e nas extremidades das folhas e pequenas manchas brancas ao longo do limbo foliar em algumas plantas das parcelas. Nos tratamentos com atrazine e nicosulfuron as plantas de milho apresentaram manchas brancas ao longo do limbo foliar e necrose nos bordos das folhas.

Para a combinação de atrazine e mesotrione os sintomas visuais de fitointoxicação foram de manchas brancas ao longo do limbo foliar. Os sintomas de fitointoxicação observados para a mistura de nicosulfuron+mesotrione foram de leve clorose ao longo das nervuras do limbo foliar em algumas plantas das parcelas.

$\mathrm{Na}$ (Tabela 3) estão apresentados os resultados de porcentagem de controle de D. horizontalis aos 26, 36, 56 e 112 DAS, para o espaçamento convencional e reduzido. Para o espaçamento convencional $(0,90$ m) todos os tratamentos herbicidas apresentaram controle satisfatório $(>80 \%)$ para capim-colchão aos 26 DAS. No entanto, os tratamentos atrazine + nicosulfuron + mesotrione, atrazine + nicosulfuron e nicosulfuron+mesotrione em aplicação única apresentaram desempenho de controle inferior a testemunha capinada, tanto aos 26 quanto aos 56 DAS. Na avaliação subseqüente (56 DAS) os tratamentos da modalidade de aplicação única tiveram redução na eficiência e os tratamentos com as misturas de atrazine+nicosulfuron e nicosulfuron + mesotrione apresentaram controle insatisfatório (respectivamente 71,25\% e 74,50\%). Para a modalidade de aplicação sequencial todos os tratamentos mantiveram níveis excelentes de controle sobre $D$. horizontalis $(\geq 94,50 \%)$.

$\mathrm{Na}$ avaliação pré-colheita (112 DAS) com exceção da mistura de atrazine+mesotrione, os demais tratamentos em aplicação única proporcionaram níveis de controle insatisfatórios $(<80 \%)$. Em contrapartida os mesmos herbicidas aplicados em modalidade sequencial mantiveram os valores de controle de $D$. horizontalis semelhantes à testemunha capinada, o que sugere que a aplicação sequencial contribui para uma maior duração do período de controle de capim-colchão. Segundo Trezzi et al. (2008), a aplicação única de atrazine+simazine em pós-emergência precoce das plantas daninhas apresentou baixa capacidade de controlar fluxos tardios das espécies Brachiaria plantaginea, Euphorbia heterophylla e Avena strigosa, havendo nova infestação na área.

Para o espaçamento reduzido aos 26 DAS todos os tratamentos apresentaram excelentes níveis de controle $D$. horizontalis $(\geq 93,50 \%)$. As combinações de atrazine + nicosulfuron + mesotrion e, atrazine + mesotrione, nicosulfuron + mesotrione em aplicação sequencial e atrazine+nicosulfuron+ mesotrione em aplicação única apresentaram controles semelhantes à testemunha capinada. Por outro lado, as misturas de atrazine+nicosulfuron tanto em aplicação única quanto em sequencial e a aplicação única de atrazine+mesotrione e nicosulfuron+mesotrione apresentaram controles inferiores à testemunha capinada.

Nas avaliações de 36, 56 e 112 DAS o agrupamento das médias seguiu um comportamento semelhante. A partir dos 36 DAS, a mistura em tanque de atrazine+nicosulfuron, independente da modalidade de aplicação, apresentou níveis de controle significativamente inferiores aos demais tratamentos, e suas médias de controle 
foram decrescendo a cada avaliação, passando a ser considerado insatisfatório a partir de 56 DAS. Os demais tratamentos mantiveram controles semelhantes à testemunha capinada até os 112 DAS. Mesmo não havendo diferença entre os tratamentos observou-se que, com exceção da mistura entre atrazine e nicosulfuron, as demais combinações de herbicidas aplicadas na modalidade sequencial mantiveram bons níveis de controle $(\geq 85 \%)$ até os 112 DAS, fato não observado na aplicação única.

Tabela 3. Porcentagem de controle de capim-colchão (Digitaria horizontalis) em quatro épocas de avaliações realizadas aos $26,36,56$ e 112 dias após a semeadura do milho cultivado em espaçamento convencional $(0,90 \mathrm{~m}) \mathrm{e}$ reduzido (0,45 m). Maringá, PR, 2008/2009.

\begin{tabular}{|c|c|c|c|c|c|c|c|c|}
\hline \multirow{4}{*}{ Tratamentos } & \multicolumn{8}{|c|}{ \% de controle de $D$. horizontalis } \\
\hline & \multicolumn{8}{|c|}{ Espaçamento } \\
\hline & \multicolumn{4}{|c|}{$0,90 \mathrm{~m}$} & \multicolumn{4}{|c|}{$0,45 \mathrm{~m}$} \\
\hline & 26 DAS & 36 DAS & 56 DAS & 112 DAS & 26 DAS & 36 DAS & 56 DAS & 112 DAS \\
\hline 1. $\mathrm{A}+\mathrm{N}+\mathrm{M}$ & $91,50 \mathrm{~b}$ & $94,75 \mathrm{a}$ & $83,75 \mathrm{~b}$ & $60,00 \mathrm{~b}$ & $99,75 \mathrm{a}$ & $96,50 \mathrm{a}$ & $97,00 \mathrm{a}$ & $80,50 \mathrm{a}$ \\
\hline 2. $\mathrm{A}+\mathrm{N}$ & $94,50 \mathrm{~b}$ & $92,50 \mathrm{~b}$ & $71,25 \mathrm{c}$ & $57,50 \mathrm{~b}$ & $96,75 \mathrm{a}$ & $88,50 \mathrm{~b}$ & $79,50 \mathrm{c}$ & $60,00 \mathrm{~b}$ \\
\hline 3. $\mathrm{A}+\mathrm{M}$ & $98,25 \mathrm{a}$ & $97,50 \mathrm{a}$ & $89,50 \mathrm{a}$ & $87,50 \mathrm{a}$ & $97,75 \mathrm{~b}$ & $98,00 \mathrm{a}$ & $97,25 \mathrm{a}$ & $79,50 \mathrm{a}$ \\
\hline 4. $\mathrm{N}+\mathrm{M}$ & 90,75 b & $93,25 \mathrm{~b}$ & $74,50 \mathrm{c}$ & $59,50 \mathrm{~b}$ & $93,50 \mathrm{c}$ & $94,00 \mathrm{a}$ & $91,50 \mathrm{~b}$ & $69,50 \mathrm{a}$ \\
\hline 5. $\mathrm{A}+\mathrm{N}+\mathrm{M} / \mathrm{A}+\mathrm{N}+\mathrm{M}$ & $99,75 \mathrm{a}$ & $97,75 \mathrm{a}$ & $98,50 \mathrm{a}$ & $91,00 \mathrm{a}$ & $100,00 \mathrm{a}$ & $98,00 \mathrm{a}$ & $98,75 \mathrm{a}$ & $90,50 \mathrm{a}$ \\
\hline $6 . \mathrm{A}+\mathrm{N} / \mathrm{A}+\mathrm{N}$ & $99,50 \mathrm{a}$ & $98,75 \mathrm{a}$ & $95,50 \mathrm{a}$ & $80,50 \mathrm{a}$ & $97,75 \mathrm{~b}$ & $89,75 \mathrm{~b}$ & $78,75 \mathrm{c}$ & $47,00 \mathrm{~b}$ \\
\hline 7. $\mathrm{A}+\mathrm{M} / \mathrm{A}+\mathrm{M}$ & $100,00 \mathrm{a}$ & $97,50 \mathrm{a}$ & $97,50 \mathrm{a}$ & $95,50 \mathrm{a}$ & $100,00 \mathrm{a}$ & $98,25 \mathrm{a}$ & $99,25 \mathrm{a}$ & $92,00 \mathrm{a}$ \\
\hline 8.N+M/N+M & $99,75 \mathrm{a}$ & $98,25 \mathrm{a}$ & $94,50 \mathrm{a}$ & $89,50 \mathrm{a}$ & $100,00 \mathrm{a}$ & $98,25 \mathrm{a}$ & $98,50 \mathrm{a}$ & $85,00 \mathrm{a}$ \\
\hline 9.testemunha sem capina & $0,00 \mathrm{c}$ & $0,00 \mathrm{c}$ & $0,00 \mathrm{~d}$ & $0,00 \mathrm{c}$ & $0,00 \mathrm{~d}$ & $0,00 \mathrm{c}$ & $0,00 \mathrm{~d}$ & $0,00 \mathrm{c}$ \\
\hline 10.testemunha capinada & $100,00 \mathrm{a}$ & $100,00 \mathrm{a}$ & $100,00 \mathrm{a}$ & $100,00 \mathrm{a}$ & $100,00 \mathrm{a}$ & $100,00 \mathrm{a}$ & $100,00 \mathrm{a}$ & $100,00 \mathrm{a}$ \\
\hline $\mathrm{F}$ & $122,01 *$ & $413,16^{*}$ & $73,48^{*}$ & $20,51^{*}$ & $955,75^{*}$ & $711,84^{*}$ & $393,68^{*}$ & $18,17^{*}$ \\
\hline $\mathrm{CV}(\%)$ & 6,40 & 3,39 & 8,70 & 18,28 & 2,28 & 2,66 & 3,67 & 19,57 \\
\hline
\end{tabular}

Obs: $\mathrm{A}=$ atrazine, $\mathrm{N}=$ nicosulfuron, $\mathrm{M}=$ mesotrione.

Médias na mesma coluna seguidas de mesma letra não diferem pelo teste de Scott-Knott a 5\% de probabilidade.

Segundo Oliveira Júnior et al. (2006a) há uma tendência consistente de que as aplicações sequenciais proporcionem controle mais efetivo das plantas daninhas. Tal evidência explica-se pelo fato de que na aplicação sequencial existe a possibilidade de controlar fluxos mais tardios de infestação, além de reforçar o controle obtido pela primeira aplicação em espécies de difícil controle, aumentando assim a eficiência de controle.

Os resultados da avaliação do controle do total de plantas daninhas na fase de pré-colheita estão descritos na (Tabela 4). Para a cultura conduzida em espaçamento convencional demonstrou que todos os tratamentos da aplicação sequencial mantiveram níveis de controle satisfatórios e semelhantes à testemunha capinada mesmo no final do ciclo da cultura, já os tratamentos com aplicação única proporcionaram controle insatisfatório nesta avaliação, com exceção da mistura de atrazine + mesotrione $(86,75 \%$ de controle $)$. De forma geral, os tratamentos em aplicação única proporcionaram controle geral médio de $73,88 \%$ e os tratamentos aplicados na modalidade sequencial obtiveram controle médio de $87 \%$. 
Tabela 4. Porcentagem de controle do total de infestantes na fase de pré-colheita da cultura do milho cultivada em espaçamento convencional (0,90 m) e reduzido (0,45 m). Maringá, PR, 2008/2009.

\begin{tabular}{|c|c|c|}
\hline \multirow{3}{*}{ Tratamentos } & \multicolumn{2}{|c|}{$\%$ de controle geral } \\
\hline & \multicolumn{2}{|c|}{ Espaçamentos } \\
\hline & $0,90 \mathrm{~m}$ & $0,45 \mathrm{~m}$ \\
\hline 1. $A+N+M$ & $75,00 \mathrm{~b}$ & $77,50 \mathrm{a}$ \\
\hline 2. $\mathrm{A}+\mathrm{N}$ & $67,50 \mathrm{~b}$ & $46,25 \mathrm{~b}$ \\
\hline 3. $\mathrm{A}+\mathrm{M}$ & $86,75 \mathrm{a}$ & $78,00 \mathrm{a}$ \\
\hline 4. $\mathrm{N}+\mathrm{M}$ & $66,25 \mathrm{~b}$ & $64,25 \mathrm{a}$ \\
\hline Média & 73,88 & 66.50 \\
\hline $5 . \mathrm{A}+\mathrm{N}+\mathrm{M} / \mathrm{A}+\mathrm{N}+\mathrm{M}$ & $89,50 \mathrm{a}$ & $90,00 \mathrm{a}$ \\
\hline $6 . \mathrm{A}+\mathrm{N} / \mathrm{A}+\mathrm{N}$ & $83,75 \mathrm{a}$ & $41,25 \mathrm{~b}$ \\
\hline 7. $\mathrm{A}+\mathrm{M} / \mathrm{A}+\mathrm{M}$ & $89,50 \mathrm{a}$ & $92,50 \mathrm{a}$ \\
\hline 8.N+M/N+M & $85,25 \mathrm{a}$ & $80,25 \mathrm{a}$ \\
\hline Média & 87 & 76 \\
\hline 9.testemunha sem capina & $0,00 \mathrm{c}$ & $0,00 \mathrm{c}$ \\
\hline 10.testemunha capinada & $100,00 \mathrm{a}$ & $100,00 \mathrm{a}$ \\
\hline $\mathrm{F}$ & $35,84^{*}$ & $14,54^{*}$ \\
\hline CV $(\%)$ & 12,64 & 23,72 \\
\hline
\end{tabular}

Obs: $\mathrm{A}=$ atrazine, $\mathrm{N}=$ nicosulfuron, $\mathrm{M}=$ mesotrione.

Médias na mesma coluna seguidas de mesma letra não diferem pelo teste de Scott-Knott a 5\% de probabilidade.

Já para o espaçamento reduzido os únicos tratamentos que apresentaram controles significativamente inferiores à testemunha capinada foi a combinação de atrazine+nicosulfuron aplicada nas modalidades única e sequencial $(46,25 \%$ e $41,25 \%$, respectivamente). Embora, não significativa a aplicação sequencial dos herbicidas atrazine+ nicosulfuron + mesotrione, atrazine + mesotrione e nicosulfuron + mesotrione se destacaram pelos elevados percentuais de controle (média de 87 e76\%). É provável que estes resultados se devam ao fato das plantas daninhas estarem em estádios iniciais de desenvolvimento no momento das aplicações sequenciais, de forma geral os herbicidas são mais efetivos no controle de plantas daninhas em estádios iniciais de desenvolvimento.

A aplicação sequencial de herbicidas se destaca por proporcionar maior percentual de controle de plantas daninhas comparado com a aplicação única. Neste sentido Oliveira Júnior et al. (2006 b) relataram que a aplicação sequencial do herbicida flumiclorac-pentil em pós-emergência precoce de plantas de Euphorbia heterophylla apresentou melhores resultados que a aplicação única. Burke et al. (2008) também observaram que a aplicação sequencial do herbicida nicosulfuron em pósemergência foi mais eficiente no controle de plantas daninhas na cultura do milho que a aplicação única. Segundo Trezzi et al. (2008) a aplicação sequencial de atrazine+simazine em pós-emergência precoce complementada por uma aplicação em pósemergência tardia de atrazine+nicosulfuron foram eficientes no controle de plantas daninhas, e isto se refletiu em uma menor produção de matéria seca das plantas daninhas, comparado com os tratamentos aplicados na modalidade única em pós-emergência precoce. Vale destacar de que a aplicação sequencial de herbicidas em pós-emergência pode ser uma ferramenta importante no controle de plantas daninhas de difícil controle, como Euphorbia heterophylla.

Desta forma, a aplicação sequencial pode ser uma opção interessante a cultura do milho, pois possibilitou a manutenção de níveis excelentes de controle para as plantas daninhas avaliadas durante a fase em que a cultura é mais sensível à competição. 
O controle se manteve até a fase de pré-colheita, o que pode proporcionar alguns benefícios, como facilitar a operação de colheita mecanizada e beneficiar a qualidade do produto colhido, ou até mesmo ser uma alternativa para redução dos níveis de infestação e adição de sementes ao solo em áreas de alta infestação facilitando, desta forma, o manejo de plantas daninhas na cultura subseqüente.

Na Tabela 5 estão apresentados os resultados dos componentes da produtividade número de espigas por hectare, peso médio das espigas e relação peso dos grãos peso total para os espaçamentos convencional e reduzido. Não houve diferença significativa entre os diferentes tratamentos para as variáveis-resposta número de espigas por hectare e peso médio da espiga, para ambos os espaçamentos. Nota-se que, de forma geral, para o espaçamento convencional houve um menor número de espigas por hectare comparativamente com os resultados em espaçamento reduzido, em média 50,18 no espaçamento de $0,90 \mathrm{~m}$ contra 55,89 no espaçamento de $0,45 \mathrm{~m}$. Porém, o peso médio de espiga foi superior no espaçamento convencional com valores médio de 215,47 gramas por espiga, contra 200,77 para o espaçamento reduzido. Já para relação peso de grãos peso total a única diferença significativa foi encontrada no tratamento de atrazine+mesotrione em aplicação única para o espaçamento de 0,90 m, sendo esta relação superior as demais.

Tabela 5. Efeito dos tratamentos sobre o número de espigas - NE, peso médio da espiga- PME, razão massa de grãos/ massa total das espigas - RGMT e rendimento de grãos - PROD para a cultura do milho cultivada em espaçamento convencional (0,90 m) e reduzido (0,45 m). Maringá, PR, 2008/2009.

\begin{tabular}{|c|c|c|c|c|c|c|c|c|}
\hline \multirow[t]{4}{*}{ Tratamentos } & \multicolumn{8}{|c|}{ Componentes da produtividade } \\
\hline & \multicolumn{8}{|c|}{ Espaçamento } \\
\hline & \multicolumn{3}{|c|}{$0,90 \mathrm{~m}$} & \multicolumn{5}{|c|}{$\mathbf{0 , 4 5} \mathrm{m}$} \\
\hline & $\begin{array}{c}\mathbf{N E} \\
\left(\mathrm{mil} \mathrm{ha}^{-1}\right)\end{array}$ & $\begin{array}{c}\text { PME } \\
(\mathrm{g})\end{array}$ & RGMT & $\begin{array}{c}\text { PROD } \\
\left(\mathrm{kg} \mathrm{ha}^{-1}\right)\end{array}$ & $\begin{array}{c}\text { NE } \\
\left(\mathrm{mil} \mathrm{ha}^{-1}\right)\end{array}$ & $\begin{array}{c}\text { PME } \\
(\mathrm{g})\end{array}$ & RGMT & $\begin{array}{c}\text { PROD } \\
\left(\mathrm{kg} \mathrm{ha}^{-1}\right)\end{array}$ \\
\hline 1. $\mathrm{A}+\mathrm{N}+\mathrm{M}$ & $49,03 \mathrm{a}$ & $216,94 \mathrm{a}$ & $0,6706 \mathrm{~b}$ & $7288,87 \mathrm{~b}$ & $55,69 \mathrm{a}$ & $199,62 \mathrm{a}$ & $0,6812 \mathrm{a}$ & $7743,54 \mathrm{a}$ \\
\hline 2. $\mathrm{A}+\mathrm{N}$ & $49,03 \mathrm{a}$ & $211,20 \mathrm{a}$ & $0,6467 \mathrm{~b}$ & $6866,40 \mathrm{~b}$ & $59,86 \mathrm{a}$ & $203,03 \mathrm{a}$ & $0,6465 \mathrm{a}$ & $7129,33 \mathrm{~b}$ \\
\hline 3. $\mathrm{A}+\mathrm{M}$ & $50,97 \mathrm{a}$ & $215,77 \mathrm{a}$ & 0,7470 a & $8401,24 \mathrm{a}$ & 54,31 a & $211,42 \mathrm{a}$ & $0,6808 \mathrm{a}$ & $7970,87 \mathrm{a}$ \\
\hline 4.N+M & $50,14 \mathrm{a}$ & $221,25 \mathrm{a}$ & $0,6631 \mathrm{~b}$ & $7530,41 \mathrm{~b}$ & 58,89 a & $196,51 \mathrm{a}$ & $0,6732 \mathrm{a}$ & $7843,00 \mathrm{a}$ \\
\hline 5. $\mathrm{A}+\mathrm{N}+\mathrm{M} / \mathrm{A}+\mathrm{N}+\mathrm{M}$ & $51,53 \mathrm{a}$ & $213,60 \mathrm{a}$ & $0,6572 b$ & $7374,12 b$ & 57,08 a & $196,67 \mathrm{a}$ & $0,6807 \mathrm{a}$ & $7757,75 \mathrm{a}$ \\
\hline $6 . \mathrm{A}+\mathrm{N} / \mathrm{A}+\mathrm{N}$ & $49,72 \mathrm{a}$ & $214,70 \mathrm{a}$ & $0,6554 \mathrm{~b}$ & $7132,58 \mathrm{~b}$ & $54,17 \mathrm{a}$ & $209,80 \mathrm{a}$ & $0,6768 \mathrm{a}$ & $7871,42 \mathrm{a}$ \\
\hline 7.A+M/A+M & $48,33 \mathrm{a}$ & $248,65 \mathrm{a}$ & $0,6427 \mathrm{~b}$ & $7843,00 \mathrm{a}$ & $54,86 \mathrm{a}$ & $195,81 \mathrm{a}$ & $0,6762 \mathrm{a}$ & $7695,17 \mathrm{a}$ \\
\hline 8.N+M/N+M & $49,58 \mathrm{a}$ & $209,58 \mathrm{a}$ & $0,6678 \mathrm{~b}$ & $7033,12 \mathrm{~b}$ & $55,42 \mathrm{a}$ & $199,90 \mathrm{a}$ & $0,6748 \mathrm{a}$ & $7629,87 \mathrm{a}$ \\
\hline 9.testemunha sem capina & $51,39 \mathrm{a}$ & $188,25 \mathrm{a}$ & $0,6789 \mathrm{~b}$ & $5592,62 \mathrm{c}$ & $51,94 \mathrm{a}$ & $194,16 \mathrm{a}$ & $0,6662 \mathrm{a}$ & $6398,96 \mathrm{~b}$ \\
\hline 10.testemunha capinada & $53,06 \mathrm{a}$ & $214,80 \mathrm{a}$ & $0,6344 \mathrm{~b}$ & $7416,75 \mathrm{~b}$ & 56,67 a & $200,82 \mathrm{a}$ & $0,6730 \mathrm{a}$ & $7899,83 \mathrm{a}$ \\
\hline $\mathrm{F}$ & $0,22^{\text {ns }}$ & $2,23^{\text {ns }}$ & $2,25^{*}$ & $6,36^{*}$ & $0,37^{\text {ns }}$ & $0,38^{\text {ns }}$ & $1,34^{\mathrm{ns}}$ & $2,79^{*}$ \\
\hline CV (\%) & 12,19 & 9,13 & 6,27 & 7,72 & 13,49 & 9,43 & 2,66 & 7,68 \\
\hline
\end{tabular}

Obs: $\mathrm{A}=$ atrazine, $\mathrm{N}=$ nicosulfuron, $\mathrm{M}=$ mesotrione.

Médias na mesma coluna seguidas de mesma letra não diferem pelo teste de Scott-Knott a 5\% de probabilidade.

NE: Número de espigas; PME:Peso médio de espigas; RGMT: razão massa de grãos/massa total das espigas; PROD: Rendimento de grãos.

O rendimento de grãos não foi afetado pela aplicação dos tratamentos quando a cultura foi semeada no espaçamento de $0,90 \mathrm{~m}$, sendo que a mistura de atrazine+mesotrione apresentou produtividades superiores a testemunha capinada independentemente da modalidade de aplicação (Tabela 5). Resultados semelhantes foram obtidos no espaçamento reduzido, no entanto, a mistura em tanque de atrazine+nicosulfuron aplicada em modalidade única apresentou redução significativa 
no rendimento de grãos provavelmente devido aos baixos valores de controle de plantas daninhas obtidos por este tratamento durante as avaliações. Mesmo que os resultados não diferiram estatisticamente, observa-se que em média o rendimento de grãos foi superior quando a cultura do milho foi conduzida em espaçamento reduzido, obtendo valores médios de $7.593,97 \mathrm{~kg} \mathrm{ha}^{-1}$, já para o espaçamento de 0,90 $\mathrm{m}$ o rendimento médio foi de $7.247,91 \mathrm{~kg} \mathrm{ha}^{-1}$.

\section{Conclusões}

Nas condições em que foi conduzido o experimento pode-se concluir que:

Os tratamentos utilizados foram eficientes no controle de $D$. horizontalis.

De forma geral, os tratamentos avaliados foram seletivos a cultura do milho e não afetaram significativamente os componentes de produção e o rendimento de grãos.

Os resultados de controle para modalidade de aplicação sequencial mantiveram valores de controle satisfatórios, para a maioria dos tratamentos até a pré-colheita da cultura do milho, sendo um indício que esta modalidade de aplicação apresenta um período de controle efetivo maior para as misturas em tanque avaliadas.

De forma geral, a redução do espaçamento não influenciou na seletividade, na eficiência dos herbicidas e no rendimento de grãos.

\section{Referências}

ARAÚJo, M. A.; TORMENA, C. A.; SILVA, A. P. Propriedades físicas de um latossolo vermelho distrófico cultivado e sob mata nativa. Rev. Bras. Ci. Solo, Viçosa, MG, v. 28, n. 2, p. 337-345, 2004.

BALBINOT JUNIOR, A. A.; FLECK, N. G. Manejo de plantas daninhas na cultura do milho em função do arranjo espacial de plantas e características dos genótipos. Ciência Rural, Santa Maria, v. 34, n. 6, p. 245-252, 2004.

BURKE, I. C.; THOMAS, W. E.; ALLEN, J. R.; COLLINS, J.; WILCUT, J. W. A comparison of weed control in herbicide-resistant, herbicide-tolerant, and conventional corn. Weed Technol., Washington, v. 22, n. 4, p. 571-579, 2008.

CARVALHO, F. T.; PERUCHI, M.; PALAZZO, R. R. B. Eficácia de herbicidas no controle, em pós-emergência, de plantas daninhas na cultura do milho. Rev. Bras. de Herb, Brasília, v. 2, n. 3, p. 143-147, 2001.

CARVALHO, L. B.; BIANCO, S.; PITELLI, R. A.; BIANCO, M. S. Estudo comparativo do acúmulo de massa seca e macronutrientes por plantas de milho var. BR- 106 e Brachiaria plantaginea. Planta Daninha, Londrina, v. 25, n. 2, p. 293-301, 2007.

CONSTANTIN, J.; OLIVEIRA JUNIOR, R. S. de; KAJIHARA, L. H.; ARANTES, J. G. Z. de; CAVALIERI, S. D.; ALONSO, D. G. Controle de diferentes espécies de guaxuma com aplicação sequencial de flumicloracpentil. Acta Sci. Agron., Maringá, v. 29, n. 4, p. 475-480, 2007.

DEMÉTRIO, C. S.; FORNASIERI FILHO, D.; CAZETTA, J. O.; CAZETTA, D. A. Desempenho de híbridos de milho submetidos a diferentes espaçamentos e densidades populacionais. Pesq. Agropec. Bras., Brasília, v. 43, n. 2, p. 1691-1697, 2008.

EMPRESA BRASILEIRA DE PESQUISA AGROPECUÁRIA - EMBRAPA. Centro Nacional de Pesquisa de Solos (Rio de Janeiro, RJ). Sistema brasileiro de classificação de solos. Brasília: EmbrapaSPI, Embrapa-CNPS, 1999. 412 p.

EUROPEAN WEED RESEARCH COUNCIL - EWRC. Report of the $3^{\text {rd }}$ and 4rd meetings of EWRC. Committee of methods in Weed Research. Weed Res., v. 4, n. 1, p. 88, 1964.

FERREIRA, L. R.; DURIGAN, J. C. ; CHURATAMASCA, M. G. C. Seletividade de herbicidas para cebola em semeadura direta. Planta Daninha, Londrina, V. 17, n. 1, p. 53-62, 1999.

FERREIRA, L. R.; DURINGAN, J. C.; CHURATAMASCA, M. G. C.; FERREIRA, F. A.; SILVA, A. A. Seletividade e eficácia da aplicação sequencial de oxyfluorfen e de ioxyniloctanoato, em semeadura direta de cebola. Planta Daninha, Londrina, v. 18, n. 1, p. 3950, 2000.

GALON, L.; PINTO, J. J. O.; ROCHA, A. A.; CONCENÇO, G.; SILVA, A. F.; ASPIAZÚ, I.; FERREIRA, E. A.; FRANÇA, A. C.; FERREIRA, F. A.; AGOSTINETTO, D.; PINHO, C. F. Períodos de interferência de Brachiaria plantaginea na cultura do milho na região sul do Rio Grande do Sul. Planta Daninha, Viçosa, MG, v. 26, n. 4, p. 779-788, 2008. 
LÓPEZ-OVEJERO, R. F.; FANCELLI, A. L.; DOURADO-NETO, D.; GARCÍA y GARCÍA, A.;

CHRISTOFFOLETI, P. J. Seletividade de herbicidas para a cultura do milho (Zea mays) aplicados em diferentes estádios fenológicos da cultura. Planta Daninha, Viçosa, MG, v. 21, n. 3, p. 413-419, 2003.

MÔRO, F. V.; DAMIÃO FILHO, C. F. Alterações morfoanatômicas das folhas de milho submetidas à aplicação de nicosulfuron. Planta Daninha, Londrina, v. 17, n. 3, p. 331-337, 1999.

NICE, G. R. W.; BUEHRING, N. W.; SHAW, D. R. Sicklepod (Senna obtusifolia) response to shading, soybean (Glycine max) row spacing, and population in three management systems. Weed Technol., Washington, v. 15 , n. 1, p. 155-162, 2001.

OLIVEIRA JÚNIOR, R. S.; CONSTANTIN, J.; TOLEDO, R.; KAJIHARA, L. H.; STASIEVISKI, A.; PAGLIARI, P. H.; ARANTES, J. G. Z.; ALONSO, D. G.; ROSO, A. C. Aplicações seqüenciais de flumicloracpentil para o controle de Euphorbia heterophylla na cultura da soja. Acta Sci. Agron., Maringá, v. 28, n. 1, p. 115-122, 2006 b.

OLIVEIRA JÚNIOR, R. S.; CONSTANTIN, J.; COSTA, J. M.; CAVALIERI, S. D.; ARANTES, J. G. Z.; ALONSO, D. G.; ROSO, A. C.; BIFFE, D. F. Interação entre sistemas de manejo e de controle de plantas daninhas em pós-emergência afetando o desenvolvimento e a produtividade da soja. Planta Daninha, Viçosa, MG, v. 24, n. 4, p. 721-732, 2006a.
RONCHI, C. P.; SILVA, A. A. Controle de plantas daninhas em cafezais recém-implantados, com herbicidas aplicados em pós-emergência em área total. Planta Daninha, Londrina, v. 22, n. 4, p. 607-615, 2004.

SCOTT, A.; KNOTT, M. Cluster-analysis method for grouping means in analysis of variance. Biometrics, Washington, v. 30, n. 3, p. 507-512, 1974.

SOCIEDADE BRASILEIRA DA CIÊNCIA DAS PLANTAS DANINHAS - SBCPD. Procedimentos para instalação, avaliação e análise de experimentos com herbicidas. Londrina: SBCPD, 1995. 42 p.

STRIEDER, M. L.; SILVA, P. R. F.da; RAMBO, L.; BERGAMASCHI, H.; DALMAGO, G. A.; ENDRIGO, P. C.; JANDREY, D. B. CAracterísticas de dossel e rendimento de milho em diferentes espaçamentos e sistemas de manejo. Pesq. Agropec. Bras., Brasília, v. 43, n. 3, p. 309-317, 2008.

TEASDALE, J. R. Influence of narrow row/high corn population (Zea mays) on weed control and light transmittance. Weed Technol., Washington, v. 9, p. 113118, 1995.

TREZZI，M. M.; VIDAL，R. A.; KRUSE，N. D.; PRATES, M. V. B.; GUSTMAN, M. S.; NUNES, A. L.;

ARGENTA, G. Manejo químico de plantas daninhas na cultura do milho em função de características morfofisiológicas e redução de espaçamento da cultura. Planta Daninha, Viçosa, MG, v. 26, n. 4, p. 845-853, 2008.

ZAGONEL, J.; VENÂNCIO, W. S.; KUNZ, R. P. Efeito de métodos e épocas de controle de plantas daninhas na cultura do milho. Planta Daninha, Londrina, v. 18, n. 1, p. 143-150, 2000. 
